\title{
DECOMPOSITION OF A GROUP WITH A SINGLE DEFINING RELATION INTO A FREE PRODUCT ${ }^{1}$
}

\section{ABE SHENITZER}

Let $G$ be a group with generators $a_{\nu}, \nu=1, \cdots, n$. An application of any automorphism $A$ of the free group on the $a_{v}$ or, equivalently, of a sequence of $T$-transformations (defined below) maps $G$ upon an isomorphic group $G^{\prime}$. If $G$ is defined by a set of prescribed relations for the $a_{v}, G^{\prime}$ can be defined by transcribing the original relations in terms of the $A^{-1} a_{v}$. Even if $G$ is defined by a single relation, it is not known how far the set of all groups with a single defining relation and isomorphic to a given one is determined by the transformations $A$. However, Grushko's theorem [2 $]^{2}$ implies that at least the decomposibility of $G$ into a free product of two of its proper subgroups can be made obvious by applying a properly chosen $A$. We shall show that for a $G$ with a single defining relation a result of J. H. C. Whitehead [1] provides a constructive method for finding $A$ and some simple tests for the free indecomposability of $G$.

Definitions and Remarks. (1) T-transformations. By a $T$-transformation on the generators $a_{1}, \cdots, a_{n}$ of the free group $F$ $=F\left(a_{1}, \cdots, a_{n}\right)$ we mean a mapping of the form:

$$
\begin{aligned}
T a_{k} & =a_{k} \text { for some fixed } k, & 1 \leqq k \leqq n, \\
T a_{i} & =a_{i} \text { or } a_{i} a_{k}^{e} \text { or } a_{k}^{-\epsilon} a_{i} \text { or } a_{k}^{-\epsilon} a_{i} a_{k}^{\epsilon}, & i \neq k, 1 \leqq i \leqq n .
\end{aligned}
$$

The Greek superscripts denote either 1 or -1 . The symbol $a_{k}$ is referred to as the distinguished symbol for the given $T$-transformation. Whenever necessary, we shall indicate the distinguished symbol by writing $T_{a_{k}}$ rather than $T$.

(2) The symbol $T W$. The symbol $T W\left(W=W\left(a_{1}, \cdots, a_{n}\right), T\right.$ denotes a $T$-transformation on $a_{1}, \cdots, a_{n}$ ) denotes the word obtained by reducing $W\left(T a_{1}, \cdots, T a_{n}\right)$ (i.e., by deleting all $a_{v} a_{\nu}^{-1}, a_{\nu}^{-1} a_{\nu}$ in it).

(3) The symbol $L(W)$. If $W$ is a reduced word, then $L(W)$ denotes the number of symbols in $W$. We refer to this number as the length of $W$.

(4) T-reductions and level transformations. A $T$-transformation, as

Presented to the Society, April 24, 1954; received by the editors June 9, 1954.

1 This paper is part of a thesis presented to the Graduate School of Arts and Science of New York University in partial fulfillment of the requirements for the degree of Doctor of Philosophy. The author is indebted to Professor Wilhelm Magnus for his encouragement and valuable advice.

2 Numbers in brackets refer to the references at the end of the paper. 
applied to a (reduced) word $W$, is called:

a $T$-reduction if $L(T W)<L(W)$

and

a level transformation if $L(T W)=L(W)$.

(5) Internal and external T-transformations. Let $W$ be a reduced word, $W=W\left(a_{1}, \cdots, a_{n}\right)$. Regard it as a word in the symbols $a_{1}, \cdots, a_{n}, a$. If $T=T_{a_{i}}$, then $T$ is called an internal $T$-transformation with respect to $W$. If $T=T_{a}$, then $T$ is called an external $T$-transformation with respect to $W$.

(6) Active and inactive symbols; right, left, and transform symbols. Active and inactive words. Consider a $T$-transformation on the symbols $a_{1}, \cdots, a_{n}$. We call $a_{i}$ inactive if $T a_{i}=a_{i}$. We call $a_{i}$ active if $T a_{i} \neq a_{i}$. If $a_{i}^{\rho}$ is an active symbol and $T a_{i}^{\rho}=a_{i}^{\rho} a_{k}^{e}\left(T=T_{a_{k}}\right)$, we call $a_{i}^{\rho}$ a right symbol. If $T a_{i}^{\rho}=a_{k}^{-\varepsilon} a_{i}^{\rho}$, we call $a_{i}^{\rho}$ a left symbol. If $T a_{i}=a_{k}^{-\varepsilon} a_{i} a_{k}^{*}$, we call $a_{i}$ a transform (symbol). A word $W$ is said to be active (inactive) $T$ if one (none) of its symbols is active $T$.

(7) Conjugate T-transformations. Consider $W=W\left(a_{1}, \cdots, a_{n}\right)$ and let $a \neq a_{i}, 1 \leqq i \leqq n$. Let $T_{a}$ be a definite $T$-transformation on the symbols $a_{1}, \cdots, a_{n}, a$. We shall call an internal $T$-transformation $T_{a_{k}}$ on the symbols $a_{1}, \cdots, a_{n}$ conjugate to $T_{a}$ if, for $a_{i} \neq a_{k}$,

$$
\begin{aligned}
& T_{a} a_{i}^{p}=a_{i}^{p} a^{e} \text { implies } T_{a_{k}} a_{i}^{p}=a_{i}^{p} a_{k}^{\bullet}, \\
& T_{a} a_{i}=a^{-c} a_{i} a^{\bullet} \text { implies } T_{a_{k}} a_{i}=a_{k}^{-e} a_{i} a_{k}^{e}, \\
& T_{a} a_{i}=a_{i} \text { implies } T_{a_{k}} a_{i}=a_{i} .
\end{aligned}
$$

(8) Disjoint words. Two words are said to be disjoint if the symbols which occur in one of them do not occur in the other ( $a$ and $a^{-1}$ are not disjoint).

(9) Minimal words. $W=W\left(a_{1}, \cdots, a_{n}\right)$ is said to be minimal $(T)$ or, simply, minimal, if $L(T W) \geqq L(W)$ for every $T$ on $a_{1}, \cdots, a_{n}$. If $W$ is minimal with respect to all $T$-transformations on $a_{1}, \cdots, a_{n}$, then it is also minimal with respect to all $T$-transformations on a set of symbols containing the symbols $a_{1}, \cdots, a_{n}$.

(10) Use of the term "involves." If it is impossible to eliminate a symbol $a$ appearing in a word $W$ by writing $W$ cyclically and deleting all pairs $\left(a_{\nu} a_{p}^{-1}\right)^{ \pm 1}$, we say that $W$ involves $a$.

Lemma. Let $W=W\left(a_{1}, \cdots, a_{n}\right)$ be a (reduced) minimal word in $a_{1}, \cdots, a_{n}$. Assume that $W$ is nontrivial, i.e., $L(W)>1$. Let $a \neq a_{i}$, 
$i=1, \cdots, n$. Let $T_{a} a_{i} \neq a_{i}$ for at least one $a_{i}$ in $W$. Then $L\left(T_{a} W\right)$ $-L(W) \geqq 2$.

Obviously the to-be-proved increase in length of $W$ under $T$ is due to "trapped" $a$-symbols.

Proof. Note that if $L(W)>1$ and $W$ is minimal, it must contain at least two symbols of a kind, if any. For, let us assume that $W$ contains a single symbol $a_{1}$ and $W=\cdots a_{1} a_{j} \cdots, j \neq 1$. Then the $T$-transformation: $a_{1} \rightarrow a_{1} a_{\jmath}^{-1}, a_{i} \rightarrow a_{i}, i \neq 1$, decreases $L(W)$ by 1 , which contradicts the assumed minimality of $W$.

Now consider a definite $T_{a}$ such that $L\left(T_{a} W\right)=L(W)$. It is clear that $T_{a} W=W$. Also, $W$ must be a product of the form:

$$
W=\prod[(i \text { 's or } 1) \text { an } r(t \text { 's or } 1) \text { an } l(i \text { 's or } 1)] \text {, }
$$

where $i=$ inactive symbol, $r=$ right symbol, $l=$ left symbol, $t=$ transform. Let $a_{1}$ be the first right symbol in the above product. It is not difficult to see that the conjugate $T_{a_{1}}$ of $T_{a}$ (see definition (7)) applied to $W$ would result in the elimination of all $a_{1}$ symbols from $W$ without insertion of any other symbols. But this would decrease $L(W)$ which is impossible in view of the assumed minimality of $W$.

We know by now that $L\left(T_{a} W\right)-L(W) \geqq 1$. The "trapping" of an $a$-symbol in $T_{a} W$ may be effected by a right $a_{i}$, a left $a_{i}$, or a transform $a_{i}$. We know that $W$ must contain at least two such $a_{i}$ symbols. We claim that each of these $a_{i}$ symbols "traps" an $a$-symbol. We assume that this statement is false and proceed to deduce a contradiction.

We observe that every active (under $T_{a}$ ) symbol $a_{q}$ in $W$ which does not "trap" an $a$-symbol must be contained in a "block" of the form:

[right symbol (transforms or 1) left symbol].

As for the "trapping" symbol $a_{\imath}^{\rho}$ we assume, at first, that it is a right or a left symbol under $T_{a}$. Then, $W=W_{1} a_{i}^{\rho} W_{2}$, where $W_{i}=1$ or a word of the form (1) above and not both $W_{i}=1$. As before, $T_{a_{i}}$, assumed to be conjugate to $T_{a}$, applied to $W$ will eliminate all $a_{i}$ symbols in $W$ other than $a_{i}^{\rho}$ and will not introduce any new symbols in place of the eliminated symbols. This would decrease $L(W)$ by at least 1 , which is impossible.

There remains the possibility that the trapping symbol $a_{i}^{p}$ is a transform under $T_{a}$. Then

$$
W=W_{1}\left[a_{i}^{p} \text { (transforms or 1) left symbol }\right] W_{2}=W_{1} A W_{2}
$$


(3) $W=W_{1}$ [right symbol (transforms or 1) $\left.a_{i}^{p}\right] W_{2}=W_{1} \bar{A} W_{2}$.

We again emphasize the fact that not both words $W_{1}$ and $W_{2}$ can be 1 , for the left (right) symbol in (2) ((3)) must have a counterpart. In (2), $T_{a_{i}}^{\nu}$, where $T_{a_{i}}$ is assumed to be conjugate to $T_{a}$ and the value of $\nu= \pm 1$ is determined by the equation: $T_{a_{i}}^{\nu} l=a_{i}^{-\rho} l, l=$ left symbol, eliminates an $a_{i}^{p}$ in $A$ when applied to $W=W_{1} A W_{2}$. Also, $T_{a_{i}}^{\nu} W_{j}=W_{j}$, $j=1,2$. Similarly, in (3), $T_{a_{i}}^{\nu}$, where the value of $\nu$ is determined by the equation: $T_{a_{i}}^{\nu} r=r a_{i}^{-p}, r=$ right symbol, eliminates an $a_{i}^{p}$ in $\bar{A}$ when applied to $W=W_{1} \bar{A} W_{2}$. Also, $T_{a_{i}}^{\nu} W_{j}=W_{j}, j=1,2$. Thus, in both cases $L(W)$ is decreased which is impossible in view of the assumed minimality of $W$.

We now state a fundamental theorem of Whitehead (Theorem 3 in [1]): "Any two equivalent minimal sets $(T)$ are interchangeable by level $T$-transformations."

It follows immediately from this result that if $W_{1}$ and $W_{2}$ are two minimal forms of a word $W=W\left(a_{1}, \cdots, a_{n}\right)$ obtained from $W$ by means of $T$-transformations, then $L\left(W_{1}\right)=L\left(W_{2}\right)$. This fact and our lemma permit us to prove the following

Corollary. Let $W=W\left(a_{1}, \cdots, a_{n}\right)$. Let $W_{1}$ and $W_{2}$ be two minimal forms of $W$. Then $W_{1}$ and $W_{2}$ contain the same number of distinct symbols.

Proof. Note that if $T$ is a level transformation with respect to a minimal word $V=V\left(a_{1}, \cdots, a_{n}\right)$ which is active $T$, then:

(a): $T V$ is minimal (by Whitehead's theorem above);

(b): $T$ is internal with respect to $V$ (if $V$ is trivial, i.e. $L(V)=1$, this statement is obvious; if $V$ is nontrivial the statement follows from our lemma);

(c): The number of distinct symbols in $V$ equals the number of distinct symbols in $T V$ (since $T$ is both level and internal).

Our corollary is trivial if $L\left(W_{1}\right)=L\left(W_{2}\right)=1$. We may therefore assume that $L\left(W_{1}\right)=L\left(W_{2}\right)>1$. By Whitehead's theorem there exists a (finite) chain of level $T$-transformations $T_{1}, \cdots, T_{k}$ such that $T_{1} \cdots T_{k} W_{1}=W_{2}$, where $T_{i+1} \cdots T_{k} W_{1}$ may be supposed active $T_{i}$. The desired conclusion now follows immediately by induction on $k$ using the observations (a), (b), (c) in the beginning of the proof.

It follows from Grushko's theorem (cf. [2]) that: A group with $n \geqq 2$ generators and a single defining relation involving the $n$ generators can be decomposed into a free product if and only if it is possible to reduce the number of distinct generators in the left side of the defining relation by means of a suitable free automorphism on the generators. 
This result and the corollary to our lemma permit us to prove

Theorem 1. Let $G=G\left[a_{1}, \cdots, a_{n} ; R\left(a_{1}, \cdots, a_{n}\right)=1\right]$, where all. $a_{i}$ are involved in $R$. Let $H$ be the free product of an infinite cyclic group $\{a\}$ and $a$ nontrivial group $B$ with generators $b_{v} \neq a$. Then $G \simeq H$ if and only if any minimal form of $R$ contains at most $n-1$ distinct $a_{i}$ 's.

Proof. The sufficiency part of the proof is obvious. To prove the necessity of our condition we assume that $G \simeq H$ and that some minimal form of $R$ contains $n$ distinct symbols. By the corollary to our lemma every minimal form of $R$ contains $n$ distinct symbols. On the other hand, it follows from Grushko's theorem that it is possible, by applying a suitable free automorphism to the generators of $G$, to find a representation of $G$ such that the word on the left side of the defining relation associated with this representation contains at most $n-1$ symbols. Minimizing this word we obtain a minimal form of $R$ containing at most $n-1$ symbols, which contradicts the corollary to our lemma.

REMARK. If the number of generators of $G$ exceeds the number of generators involved in $R, G$ is obviously representable as a free product of the required form.

It is clear that if the left side of the defining relation associated with a certain set of generators of $G$ is minimal, then $G$ cannot be represented as a free product. We now state and prove criteria which ensure the minimality of a word $W\left(a_{1}, \cdots, a_{n}\right)$ and so the indecomposability into a free product of $G=G\left[a_{1}, \cdots, a_{n} ; W\left(a_{1}, \cdots, a_{n}\right)\right.$ $=1]$.

THEOREM 2. For a product of disjoint minimal words (see definitions (8) and (9)) to be minimal it is necessary and sufficient that each factor $W_{p}$ of the product be nontrivial (i.e., $L\left(W_{p}\right)>1$ for each $W_{p}$ ).

Proof. Let $W=W_{1} \cdots W_{m}, W_{p}$ minimal and nontrivial, $W_{p}, W_{q}$ disjoint for $p \neq q, 1 \leqq p, q \leqq m$. Consider any $T_{a}, a^{\rho}$ in $W_{i}$. Then $L\left(T_{a} W_{i}\right)-L\left(W_{i}\right) \geqq 0$. Also, if $j \neq i$, (i) $L\left(T_{a} W_{j}\right)-L\left(W_{j}\right)=0$ if $W_{j}$ does not contain symbols active $T_{a}$, (ii) $L\left(T_{a} W_{j}\right)-L\left(W_{j}\right) \geqq 2$ if $W_{j}$ contains symbols active $T_{a}$. In case (i) no deletions can take place at the junction(s) between $T_{a} W_{j}=W_{j}$ and its neighbor(s) $T_{a} W_{k}$ and its length remains fixed. In case (ii) the length of $W_{j}$ increases as a result of the application of $T_{a}$ by at least 2 and its losses, resulting from deletions at the junction(s) between $T_{a} W_{j}$ and its neighbor(s), cannot exceed 1 if $j=1$ or $m$, and they cannot exceed 2 if $1<j<m$. Now, $W=A W_{i} B, a^{\rho}$ in $W_{i}, A$ and $B$ not both 1 . Assume $A \neq 1$. If all the words in $A$ are inactive with respect to $T_{a}$, then $L\left(T_{a} A\right)-L(A)=0$, and no deletions take place between $A$ and $T_{a} W_{i}$. On the other hand, 
if at least one word in $A$ is active $T_{a}$, then $L\left(T_{a} A\right)-L(A) \geqq 2$. Similarly for $B$. Now consider $T_{a} A \cdot T_{a} W_{i} \cdot T_{a} B$. If no deletions take place at either junction, $L\left(T_{a} W\right)-L(W) \geqq 0$. If deletion takes place at the first junction, say, then the last word in $A$ must be active $T_{a}$ and $L\left(T_{a} A\right)-L(A) \geqq 2$. It is by now obvious that in any case $L\left(T_{a} W\right)-L(W) \geqq 0$, q.e.d.

As an immediate application of Theorem 2 we have:

The fundamental group of a closed surface cannot be represented as a free product.

THEOREM 3. Let all exponents in $W\left(a_{1}, \cdots, a_{n}\right)$ be $\geqq 2$. Then $W$ is minimal.

Proof. Apply a definite $T=T_{a_{1}}$, say, to $W$, which we can write as

$$
\begin{gathered}
W=W_{1}\left(a_{2}, \cdots, a_{n}\right) a_{1}^{k_{1}} W_{2}\left(a_{2}, \cdots, a_{n}\right) a_{1}^{k_{2}} \cdots \\
\cdot W_{p}\left(a_{2}, \cdots, a_{n}\right) a_{1}^{k_{p}} W_{p+1}\left(a_{2}, \cdots, a_{n}\right)
\end{gathered}
$$

(where $W_{1}$ or $W_{p+1}$ or both may be 1 ). Then

$$
T_{a_{1}} W=\left(T_{a_{1}} W_{1}\right) a_{1}^{k_{1}}\left(T_{a_{1}} W_{2}\right) a_{1}^{k_{2}} \cdots\left(T_{a_{1}} W_{p}\right) a_{1}^{k_{p}}\left(T_{a_{1}} W_{p+1}\right) .
$$

Observe that deletions, if any, can take place only at one end of $T_{a_{1}} W_{j}$ (cf. the definition of a $T$-transformation). This fact and our lemma yield immediately the desired conclusion.

It is obvious that the theorem holds in the following slightly more general form:

Let all exponents associated with a generator $a_{i}$ in $W$ be of the same sign and in absolute value $\geqq 2$. Then $W$ is minimal.

TheOREM 4. Let $W=V^{m}, V$ minimal. Then $W$ is minimal.

Proof. The result is trivial for $m=1$. Let $m=2$. If $T$ is a definite $T$-transformation, then $T\left(V^{2}\right)=(T V)(T V)$ and deletion can take place between the two bracketed words if and only if $T V$ is a transform, in which case $L(T V)-L(V) \geqq 1$. Consequently $L\left[(T V)^{2}\right]$ $-L\left(V^{2}\right) \geqq 0$, i.e., $V^{2}$ is minimal. It follows by induction on $k$ that $V^{2 k}$, $k=\mathrm{a}$ positive integer, is minimal. Using the reasoning employed in proving the case $m=2$, we can prove our result for $V^{2 k+1}$, and so for $V^{m}$.

The following theorem can be easily proved:

THEOREM 5. Let $K=K\left[a_{1}, a_{2} ; R\left(a_{1}, a_{2}\right)=1\right]$ and let $G=G\left[a, b ; b^{n}=1\right]$. 
Then, for $G \simeq K$ it is necessary and sufficient that $R$ be cyclically equivalent to $A^{n}\left(a_{1}, a_{2}\right)$ where $A\left(a_{1}, a_{2}\right)$ is a primitive element in the free group $F\left(a_{1}, a_{2}\right)$.

\section{REFERENCES}

1. J. H. C. Whitehead, On equivalent sets of elements in a free group, Ann. of Math. vol. 37 (1936).

2. A. G. Kurosh, Theory of groups, Gostekhizdat, 1944 (in Russian).

NEW YoRK UNIVERSITY

\section{A THEOREM ON COMMUTATIVE POWER ASSOCIATIVE LOOP ALGEBRAS ${ }^{1}$}

\section{LOWELl J. PAIGE}

Let $L$ be a loop, written multiplicatively, and $F$ an arbitrary field. Define multiplication in the vector space $A$, of all formal sums of a finite number of elements in $L$ with coefficients in $F$, by the use of both distributive laws and the definition of multiplication in $L$. The resulting loop algebra $A(L)$ over $F$ is a linear nonassociative algebra (associative, if and only if $L$ is a group).

An algebra $A$ is said to be power associative if the subalgebra $F[x]$ generated by an element $x$ is an associative algebra for every $x$ of $A$.

Theorem. Let $A(L)$ be a loop algebra over a field of characteristic not 2. A necessary and sufficient condition that $A(L)$ be a commutative, power associative algebra is that $L$ be a commutative group.

Proof. Assume that $A(L)$ is a commutative, power associative algebra. Clearly $L$ must be commutative and $x^{2} \cdot x^{2}=\left(x^{2} \cdot x\right) \cdot x$ for all $x$ of $A(L)$. Under the hypothesis that the characteristic of $F$ is not 2 , a linearization ${ }^{2}$ of this power identity yields

Presented to the Society, December 28, 1953; received by the editors June 2, 1954.

1 The preparation of this paper was sponsored in part by the Office of Naval Research.

2 See A. A. Albert, On the power associativity of rings, Summa Brasiliensis Mathematicae vol. 2 , no. 2 , pp. 21-32. 